had they spent almost all the time in discussion; but this would not have generated a book. S. A. BARNeTT

\section{Electrical Interfaces}

Interfacial Electrochemistry. By R. K. Ottewill, J. Lyklema and R. Parsons. (Reprinted from the Journal of Electroanalytical Chemistry and Interfacial Electrochemistry. Volume 37.) Pp. 426. (Elsevier: Amsterdam, London and New York, 1972.) Dfl. $100 ; \$ 31.25$.

The Electrical Double Layer. By M. J. Spaarnay. (The International Encyclopedia of Physical Chemistry and Chemical Physics. Topic 14: Properties of Interfaces. Volume 4.) Pp.xii +415. (Pergamon: Oxford and New York, December 1972.) $£ 6.50$.

THE study of interfaces is necessarily a demanding process. The number of phases and the number of components are often large and the simplest formulation becomes complex. The thickness of the interface may be vanishingly small and is likely to be bounded by zones of considerable anisotropy. Moreover, the interface invariably concentrates many otherwise minor impurities and its experimental investigation is no less difficult than its theoretical formulation. Indeed, from both points of view the physics and chemistry of interfaces presently lack much of the elegance, simplicity and certainty of, say, spectroscopy and if it is true that most searchers prefer to look where the light is, then it is not surprising to note that the study of interfaces lags considerably in its range of specific instrumentation and in its rate of progress.

This is so in spite of the importance of interfacial studies to almost all systems of practical significance including, for example, those involving reacting solids, semiconductors, enzymes and other heterogeneous catalysts, nerve cells, as well as all foodstuffs both growing and eaten. It is therefore fortunate that there remain groups of researchers actively investigating the fundamentals of interfacial phenomena and there are now signs of large strides about to be taken as the result of applying spectroscopic and other optical techniques to these systems. However, our present understanding rests firmly on thermodynamic methods and it is interesting to note the large contribution made to this branch of physical chemistry by the Dutch schools. It may be fanciful to suppose that the analytical discipline appeals to the Dutch temperament, although it is certain that their broad and extended academic education fits them well for these interdisciplinary researches. Perhaps one should also not overlook the peculiarly close relation between Dutch universities and Dutch industry, especially Philips of Eindhoven. Whatever the cause, it is certain that much of our present understanding of interfaces and particularly those of the solid-solution interface stems from Dutch laboratories and in the beginning from the guiding hand of Professor Overbeek. His delineation of colloidal behaviour was a milestone in the progress of this ancient but hitherto qualitative science and one of the books under consideration here is a collection of some thirty-eight papers on interfacial phenomena by students and colleagues of Overbeek, most of them, but by no means all, still working in Holland. This impressive set of papers was presented to Overbeek to mark the twenty-fifth anniversary of his appointment as professor at Utrecht and they deal with topics close to his interests, i.e. with double layer effects, electrokinetic phenomena, emulsions, membranes and some biological systems. It is a most useful summary of the present situation and deserves to be widely read.

The second book reviewed here is by one of Overbeek's most distinguished students, M. J. Spaarnay, with whom in 1957 he undertook the direct measurement of Van der Waals' forces. Spaarnay is well known for his thoughtful and solid contributions to the field and this is reflected in his book. It is a thorough and classical treatment of the electrical double layer (1) at the mercury-aqueous interface, (2) at the silver iodide-aqueous interface, (3) in the presence of surfactants, and (4) at semiconductor surfaces. The first of these includes a particularly valuable account of the inner part of the double layer and the second deals with a topic invariably overlooked in electrochemical texts. There is an introductory chapter of some 60 pages describing the thermodynamics of interphases and there is an epilogue containing useful generalizations on aspects still to be resolved. Apart from one or two ambiguities the text is clear and well set out. It is almost everywhere an authoritative account and each chapter is impressively supported by an extensive and recent set of references. There is only occasional reference to experimental procedures and to actual results because the stated aim is to set out the foundations of the subject and this is done here as well as anywhere. As is often the case rigour is achieved at the expense of easy understanding and this is not a book for beginners.

It also remains throughout a classical book. Notwithstanding the molecular dimensions of the interface and the importance of short range forces, the interface is firmly subjected to the concepts of time and space averaging, which ultimately exclude the important fine detail of molecular behaviour in the interface including the dynamic properties. The way out of these restrictions is experimentally through spectro- scopic studies and theoretically through the advances in the statistical mechanics of liquids, especially those based on numerical methods. These ways forward are not evident in either of these books because they are as yet speculative and possibly far from success. Both volumes, however, describe well the present level of achievement and are strongly recommended to all those actively investigating interfacial phenomena. Graham Hills

\section{Perils of the Embryo}

Drugs and Fetal Development. Edited by Marcus A. Klingberg, Armand Abramovici and Juan Chemke. (Proceedings of and International Symposium on the Effect of Prolonged Drug Usage on Fetal Development, held at Kfar Saba, Israel, September 1971.) Pp. xiv +559. (Plenum: New York and London, 1972.) \$32.

THis book is an edited account of the proceedings of an international symposium held in Israel in the autumn of 1971. It comprises some forty-four papers on a variety of topics relating to the central theme, some being reviews, some reports on original work, some essays on methodology. The reader is spared both the profundities and banalities of whatever discussion followed each communication, although presumably stimulation of discussion was the motive for bringing the participants together, and without it the material presented lacks coherence.

The symposium proper opens with an erudite historical introduction by $\mathrm{Dr}$ Warkany (the doyen of developmental pathologists) entitled "Congenital Malformations through the Ages". Subsequent contributions deal with both experimental and clinical observations, beginning with a review of reported adverse effects of drugs on the human foetus by Shirkey, who cites over 100 references, and continuing with a number of useful papers in which the significance and relevance of animal studies to possible effects on the human foetus are critically discussed. A further paper by Rennert goes into the mechanisms by which some agents affect protein synthesis in the embryo and is followed by interesting observations by Keeler on the teratogenic effect on grazing animals of certain poisonous plants and by the Lutzes on the effects of pesticides in inhibiting Müllerian suppression in bird embryos. Subsequent contributions include the inevitable description of Minamata disease by Murakami, an important paper by Laron on the foetal actions of growth hormone, and a disturbing study reported by Richards on possible effects on the foetus of salicylates taken during pregnancy. It is reassuring that Ravid and Toaff were not able to show any such effects from 